Mater. Res. Soc. Symp. Proc. Vol. 1359 (C) 2011 Materials Research Society

DOI: 10.1557/opl.2011.1016

\title{
Investigations into Current Modulation Mechanisms in Low Operating Voltage Organic Thin Film Transistors and Their Relationship to the Materials Employed
}

\author{
Daniel Elkington ${ }^{1}$, Xiaojing Zhou ${ }^{1}$, Warwick Belcher ${ }^{1}$, Paul Dastoor ${ }^{1}$ \\ ${ }^{1}$ Centre for Organic Electronics, Department of Physics, The University of Newcastle, University \\ Drive, Callaghan, NSW 2308, Australia
}

\begin{abstract}
Systematic studies have been conducted on the electrical characteristics of poly(3hexylthiophene)-based organic thin film transistors (OTFTs). The OTFTs have been characterized at low-operating voltages and deductions have been made regarding the current modulation mechanisms involved. Irreproducibility of transfer characteristics in these devices beyond a certain gate voltage, as well as a slow time-dependant component to drain current at certain gate voltages, indicates electrochemical changes occurring in the device during operation. It is hoped that this work can help to improve the understanding of OTFTs of this type and, in turn, their performance in the future.
\end{abstract}

\section{INTRODUCTION}

OTFTs which operate at low voltages have the advantage of combining all the benefits of organic electronics, such and solution processability and potential low cost, without the impractical high operating voltages required by many organic transistors (sometimes several tens of volts) [1]. They could be particularly useful as an electronic transducer in applications where sensitivity at low voltages is paramount to successful performance, such as biosensors.

Sandberg et al.[2,3], introduced the concept of a low-operating voltage OTFT with a hygroscopic insulator - poly(vinylphenol) (PVP) - which was labelled a HIFET (hygroscopic insulator field effect transistor). Here, we have further investigated similar devices with a view to understanding more clearly the mechanisms involved in current modulation.

There are several other varieties of organic transistors which operate at low voltages, frequently with better electrical performance than the devices presented here, however their fabrication typically involves processes such as thermal evaporation, thermal cross-linking or some critical inorganic components $[4,5]$. The aim of researching the proceeding types of devices lies in the desire to fabricate all-solution-processed OTFTs in a low-energy process in air.

\section{EXPERIMENTAL DETAILS}

Devices are fabricated on pre-patterned indium-tin-oxide (ITO)-on-glass substrates. Device architecture and the materials used are shown in Figure 1. ITO forms the source and drain electrodes and the channel length is $20 \mu \mathrm{m}$ while the channel width is $3 \mathrm{~mm}$. P3HT (from Lumtec) is spin-cast onto these substrates at 2000rpm for 60 seconds from chloroform solution. This layer is then manually patterned and the device is treated at $45^{\circ} \mathrm{C}$ for 10 minutes to remove 
any excess solvent. The PVP (from Sigma-Aldrich) layer is deposited from ethanol solution by the same spin conditions and the layer is again manually patterned. The device then is treated at $85^{\circ} \mathrm{C}$ for 10 minutes - again to remove any excess solvent. Finally, the PEDOT:PSS gate is drop-cast from an aqueous suspension (as received Celvios-P from Heraeus), which is allowed to dry at $45^{\circ} \mathrm{C}$.

Device characterization was performed by sweeping drain voltage $\left(V_{D}\right)$ for a given gate voltage $\left(V_{G}\right)$, switching $V_{G}$ and performing the $V_{D}$ sweep again. This process is repeated until measurements have been made for the desired range of $V_{G}$. The drain current $\left(I_{D}\right)$ was measured for each combination of $V_{G}$ and $V_{D}$. All voltages are relative to the source electrode. A typical $I_{D}-V_{D}$ characteristic of the devices is shown in Figure 2.

Experiments in the time domain were carried out by holding $V_{D}$ constant, and incrementing $V_{G}$ in steps whilst measuring $I_{D}$. The $I_{D}$ sampling frequency was approximately $10 \mathrm{~Hz}$.

In both experiments, two Keithley 2400 SourceMeters were controlled by a custom Labview program to both control $\mathrm{V}_{\mathrm{D}}$ and $\mathrm{V}_{\mathrm{G}}$ as well as measure $\mathrm{I}_{\mathrm{D}}$. All measurements were conducted in air - temperature and humidity were not controlled.

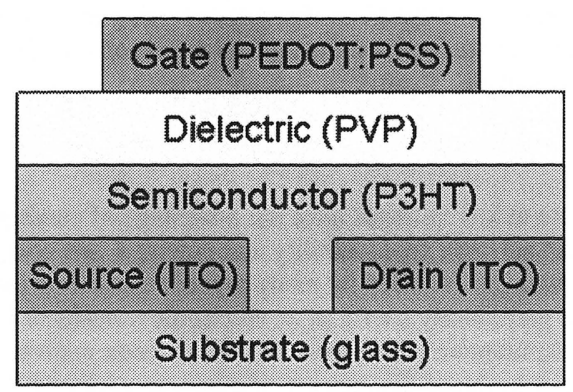

Figure 1: Device architecture and materials 


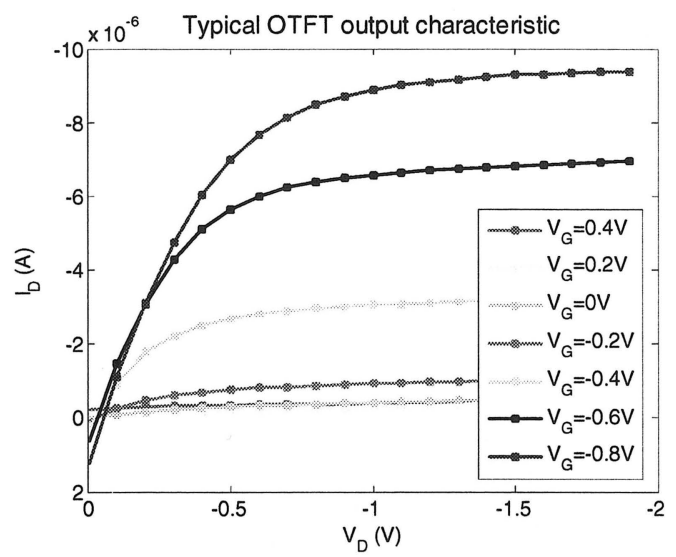

Figure 2: Representative output IV characteristic

\section{DISCUSSION}

\section{Fabrication process}

Aside from the pre-patterned ITO source and drain electrodes, the device is entirely solution processed and the only heating steps involved are low-temperature (below $100^{\circ} \mathrm{C}$ ) solvent removal steps. This supports the philosophy of moving towards an "all solution in air" style fabrication process. Using an additive, solution-based method to fabricate all parts of the device (including the source and drain electrodes) such as inkjet printing is a future objective.

\section{Transfer characteristic degradation}

When measuring the output characteristic of an OTFT, it was observed that the extrapolated transfer characteristic was systematically degraded on subsequent measurements of a single device resulting in a lower current modulation ratio $\left(\mathrm{I}_{\mathrm{ON}} / \mathrm{I}_{\mathrm{OFF}}\right)$ in each subsequent scan as shown in Figure $3 \mathrm{a}$. In contrast, if $\mathrm{V}_{\mathrm{G}}$ is restricted to scan to $-0.4 \mathrm{~V}$, highly reproducible behavior is observed shown in Figure $3 \mathrm{~b}$. If the current modulation in the devices was due entirely to an electric field or similar highly reversible effect, there is no explanation for this type of gross change in device performance from measurement-to-measurement. However, since electrochemical doping of the $\mathrm{P} 3 \mathrm{HT}$ is a likely candidate for a current modulation mechanism in these types of devices [2], we can infer that some electrochemical action is taking place when $V_{G}$ reaches some level more negative than $-0.4 \mathrm{~V}$, which changes the electrical properties of the device. Possibly, this is in the form of doping of the P3HT layer until a $\mathrm{V}_{\mathrm{G}}$ of about $-1.0 \mathrm{~V}$ and then some other de-doping or over-oxidation process at $\mathrm{V}_{\mathrm{G}}$ more negative than $-1.0 \mathrm{~V}$. The exact nature of this needs further investigation, however $\mathrm{H}^{+}$and/or $\mathrm{OH}^{-}$ions from the electrolysis of water attracted by the hygroscopic dielectric, as well as ions contributed by the PEDOT:PSS suspension are likely candidates for involvement. 

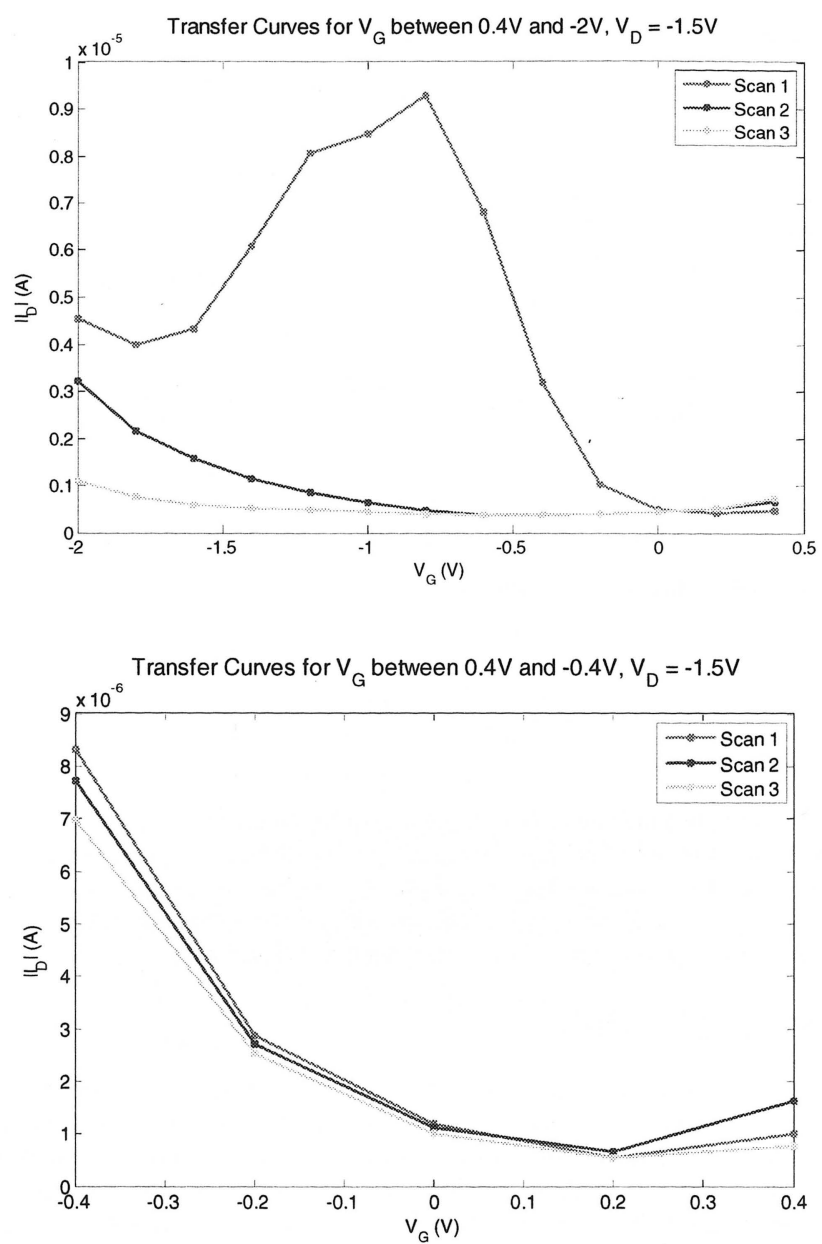

Figure 3: Transfer characteristic at $V_{D}=-1.5 \mathrm{~V}$ of three sequential IV characterizations with $\mathrm{V}_{\mathrm{G}}$ switched from (a) $0.4 \mathrm{~V}$ to $-2 \mathrm{~V}$ in $-0.2 \mathrm{~V}$ intervals and (b) $0.4 \mathrm{~V}$ to $-0.4 \mathrm{~V}$ in $-0.2 \mathrm{~V}$ intervals. 


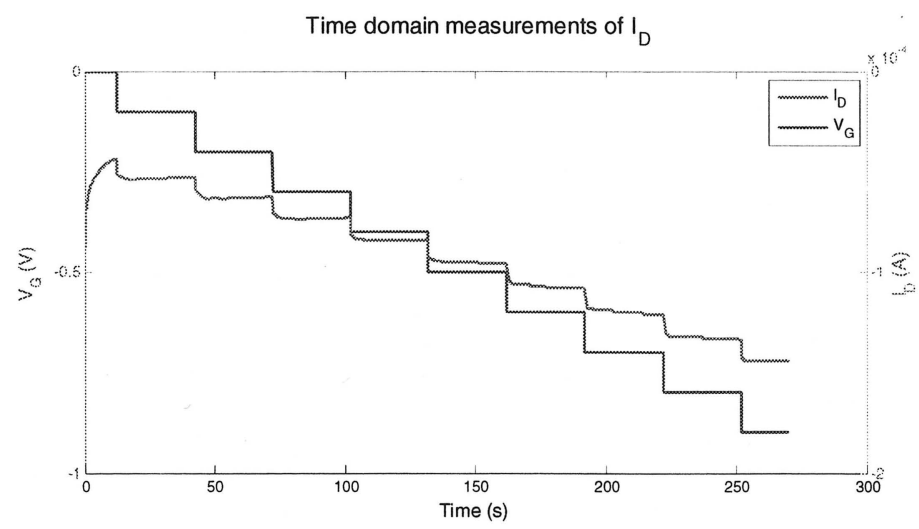

Figure 4: $I_{D}$ measurements for a fixed $V_{D}$ when $V_{G}$ is switched in $-0.1 \mathrm{~V}$ intervals (sampling frequency $\approx 10 \mathrm{~Hz}$ ).

\section{Time domain measurements}

Figure 4 depicts that once $\mathrm{V}_{\mathrm{G}}$ exceeds negative than $-0.4 \mathrm{~V}$, there is a slow, time-dependant component to the change in $I_{D}$ as well as the initial switch in the level of $I_{D}$. This is further indication of an electrochemical action taking place under these conditions since it would be expected that electrochemical changes are slow compared with the faster action of charges building up at the gate dielectric layer interfaces. It also indicates the presence of two different mechanisms contributing to the modulation of $I_{D}$, with one (charge accumulation at the dielectric's interfaces inducing an accumulation region in the semiconductor) much faster than the other (electrochemical doping of the semiconductor).

\section{CONCLUSIONS}

From the behavior of the devices in both experiments, we can make two conclusions: firstly, that there is some permanent change to the device when it is driven to $V_{G}$ beyond a threshold of $-0.4 \mathrm{~V}$; and secondly, that there are two current modulation mechanisms observed when measuring current in the time domain: a fast mechanism which we attribute to be due to charges accumulating at the interfaces of the dielectric layer, and a slower mechanism which we attribute to be electrochemical doping of the P3HT layer near the interface.

This evidence supports the conjecture of electrochemical mechanisms being involved in such devices. It is suspected that the ions which move and accumulate at the dielectric layer's interfaces as well as participate in electrochemical activity near the PVP/P3HT interface are those liberated by the attracting of water by the hygroscopic dielectric layer as well as those contributed by the PEDOT:PSS suspension. 


\section{ACKNOWLEDGMENTS}

D.E wishes to thank the University of Newcastle and the Australian Postgraduate Award Scholarship.

\section{REFERENCES}

1. Katz, H.E. and J. Huang, Thin-Film Organic Electronic Devices. Annu. Rev. Mater. Res., 2009. 39: p. 71-92.

2. Sandberg, H.G.O., et al., High Performance All Polymer Transistor Utilizing a Hygroscopic Insulator. Advanced Materials, 2004. 16(13): p. 1112-1115.

3. Sandberg, H.G.O., et al., Applications of an all-polymer solution-processed highperformance, transistor. Synthetic Metals, 2005. 155(3): p. 662-665.

4. Roberts, M.E., et al., Cross-Linked Polymer Gate Dielectric Films for Low-Voltage Organic Transistors. Chemistry of Materials, 2009. 21(11): p. 2292-2299.

5. Yang, F.Y., et al., Low-operating-voltage polymeric transistor with solution-processed low-k polymer/high-k metal-oxide bilayer insulators. Organic Electronics, 2008. 9(5): p. 925-929. 